\title{
On the Nature of Radio Eclipsing
}

\author{
Ya. N. Istomin \\ P. N. Lebedev Physical Institute, Leninsky Prospect 53, Moscow 117924, \\ Russia
}

\begin{abstract}
It is shown that the phenomena of radio eclipsing can be explained by the linear mechanism of transformation of the transverse electromagnetic wave, propagating in the pulsar wind, into the plasma waves in the region of interaction of wind with a companion star atmosphere. The coefficient of the passing $\eta$ depends on the wave frequency $\omega$ by the exponential manner $\eta=\exp \left\{-\right.$ const $\left.\cdot \omega^{-1}\right\}$. The estimated scale for the pulsar wind and star's atmosphere density gradients are of the order of 100 meters. Such gradient can be obtained in the bow shock forming when the pulsar wind enters into the companion star atmosphere. Annihilation of the part of the wind's positrons with the star's electrons produces the electric current. This current generates the magnetic field from which the pulsar wind's particles are reflected. The magnitude of the magnetic field in this shock of about several Gauss.
\end{abstract}

\section{Introduction}

The problem of the eclipsing of radio signal is that the density of the plasma in the eclipsing region is too small to explain this phenomena by any linear effect. But the nonlinear processes in the plasma of pulsar wind and the plasma of the companion star atmosphere (Thompson et al. 1994), which are proportional to the powers of the small nonlinear parameter, are less probable then the linear ones.

In this paper we discuss the linear process of transformation of transverse radio wave, frozen to the pulsar wind plasma, to the longitudinal wave in the region of interaction of the pulsar wind with the star atmosphere. We have the relativistic wind, consisting of the electrons and positrons, ejecting from the radio pulsar. The density of the electrons and positrons in the wind is $n_{w}$, the average value of it's Lorentz factor is $\gamma$, the electron density of the plasma of atmosphere is $n_{e}$. In some region we have a mixture of the wind plasma and the star's plasma. The refractive index of the plasma waves $n_{l, t} \simeq 1-\omega_{w} / \omega \gamma^{3 / 2}$ is very close to that for the electromagnetic waves $n_{t} \simeq 1-\omega_{p}^{2} / 2 \omega^{2}-\omega_{w}^{2} / 2 \omega^{2} \gamma$. In the region of interaction of the pulsar wind with the companion star atmosphere the plasma frequency of the wind $\omega_{w}=\left(8 \pi n_{w} e^{2} / m_{e}\right)^{1 / 2}$ is the falling function of the coordinate $x$ along the line of the observation, on the other hand the plasma frequency of the star's corona $\omega_{p}=\left(4 \pi n_{p} e^{2} / m_{e}\right)^{1 / 2}$ is growing function of $x$. Thus, there is the situation when the terms $n_{t}, n_{l, t}$ are crossing at some 
point. Near this point the electromagnetic wave energy transforms partially to the energy of plasma waves, damping in the atmosphere.

\section{Wave transformation and structure of the shock}

We have inhomogeneous plasma of the companion star atmosphere, described by the dielectric constant $\varepsilon=1-\delta \varepsilon, \delta \varepsilon=\omega_{p}^{2} / \omega^{2}$, through which the pulsar wind moves. The equation for the refractive index $\delta n=k c / \omega-1$ of electromagnetic waves in such matter is (Istomin \& Luk'yanov 1990)

$$
\delta n^{3}+\frac{\delta \varepsilon+\Omega_{w}^{2}}{2} \delta n^{2}-\frac{\Omega_{w}^{2}}{\gamma^{2}} \delta n-\frac{\Omega_{w}^{4}}{2 \gamma^{2}}=0, \Omega_{w}{ }^{2}=\frac{\omega_{w}^{2}}{\omega^{2} \gamma} .
$$

Using the Laplace transformation method we find the expression for the coefficient of passing $\eta$

$$
\eta=\exp \left\{-\frac{\pi}{2} \frac{\omega_{p}^{2}}{\omega c} l_{p}\left(1+\frac{l_{p}}{l_{w}}\right)\right\}
$$

Introducing the frequency of the eclipsing $\omega_{e c l}$, which means that the eclipsing exists at $\omega<\omega_{e c l}$, the scale of the plasma inhomogeneity $l \simeq l_{p} \simeq l_{w}$ can be estimated from the condition $\eta \simeq 1, l \simeq \omega_{e c l} c / \pi \omega_{p}{ }^{2}$. For J2051-0827 $\omega_{e c l} \simeq$ $2 \pi G H z, \quad \omega_{p}^{2} / \omega^{2} \simeq 4 \cdot 10^{-4}$ (Stappers et al. 1996 ) the value of $l \simeq 100 \mathrm{~m}$.

In the region where the pulsar wind interacts with the atmosphere of the companion star the bow shock appears. It is clear that the observed eclipsing takes place on the periphery of the shock far from it's forehead. We describe the structure of the shock considering that the wind's particles are stopped by the magnetic field, generating on the front. The reason of the magnetic field excitation is the annihilation of the pulsar wind's positrons in the plasma of the star's atmosphere. Although the depth with respect to annihilation $\tau_{a} \simeq$ $\sigma_{a} n_{e} l_{e c l}$ is small $\tau_{a} \simeq 10^{-7}$, it is enough to create the significant electric current $j$ because of the flux of the electrons in the pulsar wind is huge $j_{w}=e n_{w} c \simeq$ $200 \cdot \gamma^{-1}$ ampere $/ \mathrm{m}^{2}$, and the annihilation of the small part the positrons gives the noticeable current $j \simeq j_{w} \tau_{a}$.

On the periphery we can consider the shock as a plane with the width of $H$. The value of $H$ is much shorter than the scales in the transverse directions $l_{c c l}$. The solution for the shock gives the estimation $H \simeq 10^{2} \cdot \gamma^{2} \mathrm{~cm}$. The value of $H$ is just the scale of the plasma inhomogeniety in the transition region $l$. The described model of the shock does not contradict the eclipsing data if we suggest the mean Lorentz factor $\gamma$ of the pulsar wind in the vicinity of the companion star as 10 . This value is in agreement with the pulsar wind models. The magnitude of the magnetic field on the shock front is of $B \simeq 0.2 \cdot \gamma$ Gauss $\simeq 2$ Gauss.

\section{References}

Istomin, Ya.N., Luk'yanov, A.V. 1990, Sov. Phys. JETP, 70, 891.

Stappers, B.W., Bailes, M., Lyne A.G., Manchester, R.N., D'Amico, N., Tauris, T.M., Lorimer, D.R., Johnston, S., Sandhu, J.S. 1996, ApJ, 465, L119.

Thomson, C., Blandford, R.D., Evans, C.R., Phinney, E.S. 1994, ApJ, 422, 304. 